\title{
Probing the Breadth of Macrolide Glycosyltransferases: In vitro Remodeling of a Polyketide Antibiotic Creates Active Bacterial Uptake and Enhances Potency
}

\author{
Min Yang, Mark Proctor, David Bolam, James Errey, Robert Field, Harry J. Gilbert, Benjamin G. Davis*
}

\begin{abstract}
Cloning of glycosyltransferase genes into E. coli expression vectors: The plasmid vector used in this study was Miniprseta (gift from Prof. A. R. Fersht at the Medical Research Council, Cambridge, UK). Chromosomal DNA was extracted from Streptomyces lividans (DSMZ No.46482) and Streptomyces antibioticus (DSMZ No.40868) using the QIAGEN Genomic-tip 100/G kit (\#10243). The genes encoding MGT, OleD and Olel were amplified in the standard $50 \mu \mathrm{l}$ reaction mixture (advised by Novagen) containing $0.5 \mu \mathrm{g}$ of the appropriate genomic DNA, the primers listed in Table 2 and Kod Hot Start polymerase (Novagen). The PCRs were carried out using a Techne PHC-3 Thermocycler at the following temperatures; $94{ }^{\circ} \mathrm{C}$ for 2 min followed by 35 cycles at 94 ${ }^{\circ} \mathrm{C}$ for $30 \mathrm{sec}$ and $68{ }^{\circ} \mathrm{C}$ for 2 min with a final single extended elongation phase at $68{ }^{\circ} \mathrm{C}$ for $10 \mathrm{~min}$. The amplified DNA was digested with BamHI and EcoRI (Olel and MGT) or Bglll and EcoRI (OleD) and ligated into the polylinker region of Minipreseta (precut using $\mathrm{BamHI}$ and EcoRI). The ligated plasmids were transformed into the Escherichia coli strain XL1-Blue (Stratagene). The cloned genes were sequenced from two independent recombinant plasmids by MWG using the T7 promoter and T7 terminater sequencing primers (supplied by MWG). The data showed that both plasmids for each gene contained identical sequences, but differed in several places from the respective sequence deposited in GenBank. The corrected sequences have now been deposited with GenBank (OleD - Z72257, Olel - AF055579 and MGT M74717 - (original numbers)).
\end{abstract}

Additional Table 1. Oligonucleotide Primers for gene amplification

\begin{tabular}{|c|c|c|}
\hline $\begin{array}{l}\text { Target } \\
\text { gene }\end{array}$ & Upstream primer & Downstream primer \\
\hline OleD & $\begin{array}{l}5^{\prime} \text { to } 3^{\prime}- \\
\text { ATAAGATCTACC } \\
\text { ACCCAGACCAC } \\
\text { TCCCGC } \\
\text { CCACATCGCC }\end{array}$ & $\begin{array}{l}5^{\prime} \quad \text { to } 3^{\prime}- \\
\text { TAGAATTCACAAA } \\
\text { GCG } \\
\text { GATCTCTGCCGG } \\
\text { TCGGAACGG }\end{array}$ \\
\hline Olel & $\begin{array}{l}5^{\prime} \text { to } 3^{\prime}{ }^{-} \\
\text {ATAGGATCCAC } \\
\text { G } \\
\text { AGCGAGCACCG } \\
\text { CTCTGC CTCC }\end{array}$ & $\begin{array}{l}5^{\prime} \text { to } 3^{\prime} \\
\text { TAGAATTCAGCCT } \\
\text { GC } \\
\text { TTCGGCGAGGAT } \\
\text { GCCCTCC }\end{array}$ \\
\hline $\begin{array}{l}\text { S. lividans } \\
\text { MGT }\end{array}$ & $\begin{array}{l}5^{\prime} \text { to } 3^{\prime}- \\
\text { ATAGGATCCAA } \\
\text { G } \\
\text { CGAAAAGAGTT } \\
\text { GCACGAGACGT } \\
\text { CTCGTCTC }\end{array}$ & $\begin{array}{l}5^{\prime} \text { to } 3^{\prime} \\
\text { TAGAATTCAGCCG } \\
\text { CGC } \\
\text { GCGGCGGCCAGT } \\
\text { TC }\end{array}$ \\
\hline
\end{tabular}

\section{Protein expression and purification:}

The glycosyltransferases were expressed in E.coli cell strain C41(DE3) ${ }^{[1]}$ using LB medium supplemented with ampicillin at $50 \mu \mathrm{g} \mathrm{mL}^{-1}$. They were initially grown at $37^{\circ} \mathrm{C}$ to an $A_{600 \mathrm{~nm}}$ value of 0.4 before overnight induction was initiated with the addition of isopropyl- $\beta-D$ thiogalactopyranoside $(60 \mu \mathrm{M})$ at $16{ }^{\circ} \mathrm{C}(\mathrm{MGT}$ and $\mathrm{OleD})$ or $30{ }^{\circ} \mathrm{C}$ (Olel). The cells obtained from $1.6 \mathrm{~L}$ of each culture were re-suspended in $80 \mathrm{~mL}$ of $20 \mathrm{mM}$ Tris- $\mathrm{HCl}$ buffer, pH 8.0, containing $500 \mathrm{mM} \mathrm{NaCl}$ (Buffer A). The cells were disrupted by ultrasonication and the insoluble material was removed by centrifugation at $30000 \mathrm{~g}$ for 30 $\mathrm{min}$ at $4^{\circ} \mathrm{C}$. The cell-free extract was passed through 4 $\mathrm{mL}$ of TALON resin (Clontech), which was then washed with $60 \mathrm{~mL}$ of Buffer $A$ and the purified glycosyltransferases eluted in $15 \mathrm{~mL}$ of Buffer A containing $100 \mathrm{mM}$ imidazole. Purity was assessed by SDS-PAGE (Figure1) and the amounts of each protein determined using the appropriate molar-extinction coefficient (OleD is $57040 \mathrm{M}^{-1} \mathrm{~cm}^{-1}$, Olel is $44380 \mathrm{M}^{-1} \mathrm{~cm}^{-1}$ and MGT is $62730 \mathrm{M}^{-1} \mathrm{~cm}^{-1}$ ).

Addition figure 1: Preparation and purification of OleD, Olel and MGT

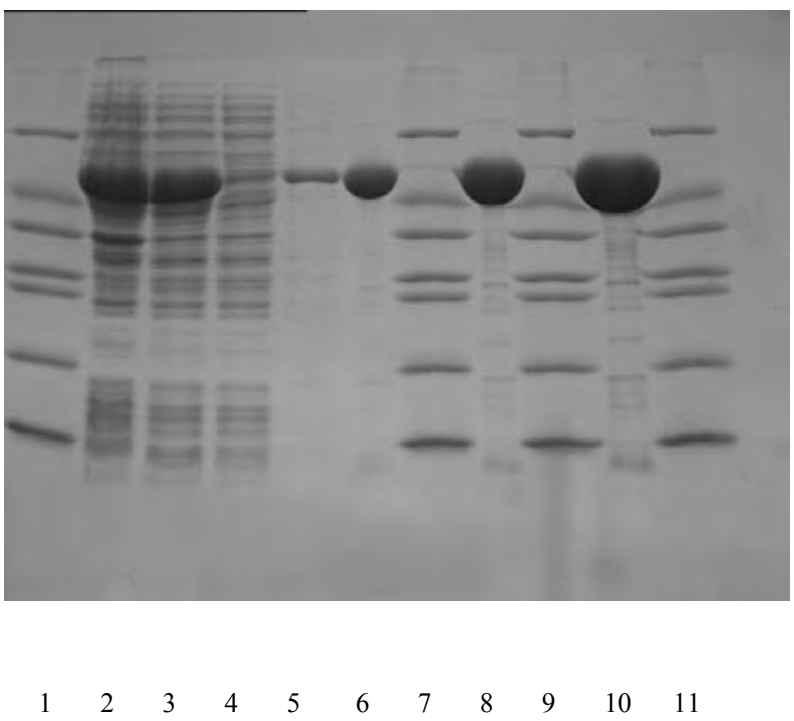

Proteins were subjected to SDS-PAGE using a $10 \%(\mathrm{w} / \mathrm{v})$ gel. The lanes contained the following samples: 1,7, 8 and 11, Dalton Mark VII-L SDS-PAGE standards (Sigma \#SDS-7; the Mr of the standard proteins are $66 \mathrm{k}, 45 \mathrm{k}, 36 \mathrm{k}$, $29 \mathrm{k}, 24 \mathrm{k}, 20$ and 14k); 2, whole cells expressing OleD; 3 , soluble cell-free extract from E. coli expressing OleD; 4, material that did not bind to the Talon column; 5, final 
wash on the Talon colum; 6, protein eluted with $100 \mathrm{mM}$ imidazole; 8, purified Olel; 10, purified MGT

Full kinetic and HTS mass spectrometric GAR screening:

Methods used taken from ref. ${ }^{[2]}$. Briefly, Waters ZMD-MS (ESl), Waters 600 HPLC system with Waters 2700 sampler were operated by Micromass Masslynx 3.3 and data processed using Masslynx 3.5, Microsoft Excel 2002 and Origin 7. The HPLC/auto-sampler control was divided into two stages: stage 1) internal standard NDP $(0.1 \mathrm{mM})$ injection (MeCN : $\mathrm{H}_{2} \mathrm{O}$ (50:50); $0.12 \mathrm{~mL} / \mathrm{min}$; isocratic for $0.1 \mathrm{~min}$; injection volume: $10.0 \mu \mathrm{L} ; 150-1000 \mathrm{ESI}^{-}$for 0.1 min, scan $0.2 \mathrm{~min}$ ); 2) 2. sample injection (MeCN : $\mathrm{H}_{2} \mathrm{O}$ (50 : 50); $0.12 \mathrm{~mL} / \mathrm{min}$; isocratic for $5.5 \mathrm{~min}$; injection volume: $10.0 \mu \mathrm{L} ; 150-1000 \mathrm{ESI}^{-}$for 5 . min, TIC of single ion peaks monitored, dwell $0.1 \mathrm{~s})$. Typical reactions: [UDPGIc] 20, 40, 60, 80, $100 \mu \mathrm{M}$; [acceptor] 20, 60, 100 $\mu \mathrm{M}$; $10 \mu \mathrm{L}$ enzyme [E] 1mg/mL; total vol $300 \mu \mathrm{LTRIS}(1 \mathrm{mM}$ , pH7.8, $1 \mathrm{mM}$ DTT). For GAR screen a 96 well plate, each well containing TRIS buffer $1 \mathrm{mM}, \mathrm{pH} 7.8,100 \mu \mathrm{l})$, donor $(10 \mathrm{mM}, 5 \mu \mathrm{l})$, acceptor $(10 \mathrm{mM}, 5 \mu \mathrm{l})$, enzymes $(1 \mathrm{mg} / \mathrm{mL}$, $5 \mu \mathrm{l})$ was incubated at $37^{\circ} \mathrm{C}$ overnight and monitored by MS (full scan from 150-1100 Da).

Addition figure 2: Illustrative mass spectra of OleD catalyzed Oleandomycin glycosylation

(a)

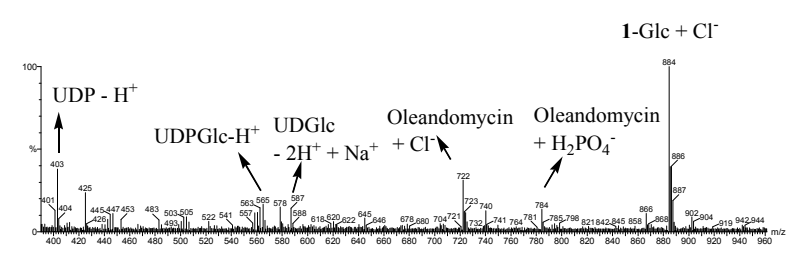

(b)

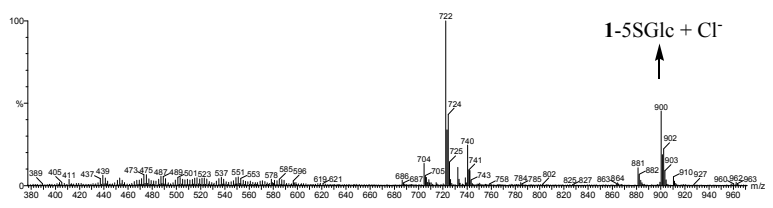

(c)

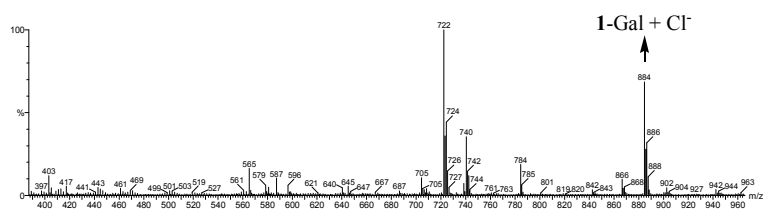

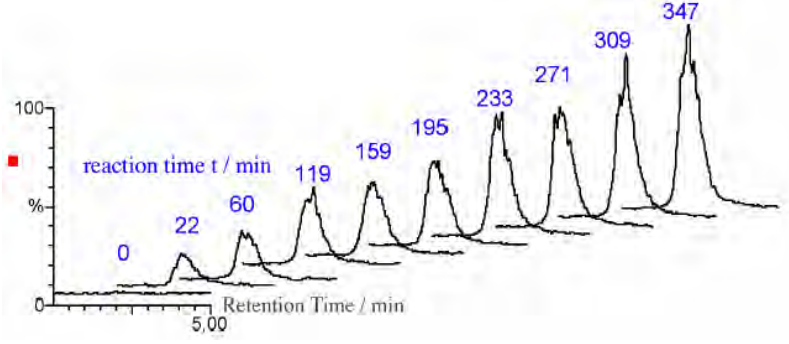

(a) using UDP-Glc + OleD: Product peaks for 1-Glc at 884, $886\left[\mathrm{M}+\mathrm{Cl}^{-}\right]^{-}$(b) using UDP-5S-Glc + OleD: 1-5S-Glc at $900,902\left[\mathrm{M}+\mathrm{Cl}^{-}\right]^{-}$(c) using UDP-Gal and Olel: 1-Gal at $884,886\left[\mathrm{M}+\mathrm{Cl}^{-}\right]^{-}$(d) total TIC time course for 1-Glc peak in (a) at [UDP-GIc] $=100 \mu \mathrm{M}$

Additional Figure 3 Lineweaver-Burke Plot for OleD with UDPGIc and Oleandomycin as substrates

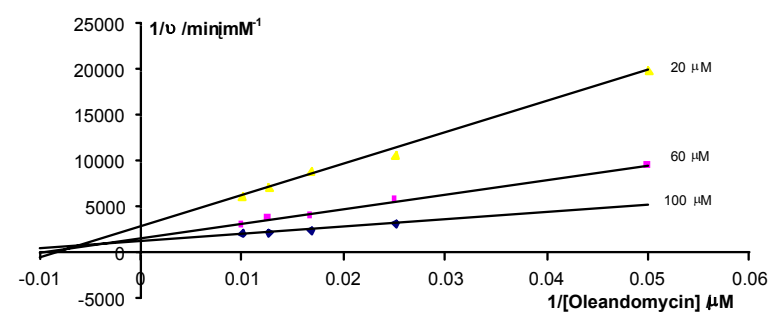

Lineweaver-Burke intersection in the $2^{\text {nd }}$ quadrant indicated that $\mathrm{K}_{\mathrm{IA}}>\mathrm{K}_{\mathrm{A}}$.

Additional Table 2 Regression with the hypothesis of Oleandomycin as $A$

Equation $Y=1 /\left(P 1 / A^{*} B /\left(P 2{ }^{*} P 4+P{ }^{*} B+P 4 / A+B / A\right)\right)$

$\begin{array}{lllll}\mathrm{V}_{\text {max }} & \mathrm{P} 1 & 0.00095 & \pm 0.00022 & \mathrm{mM} \\ \mathrm{K}_{\mathrm{l} \text { (oleandomycin) }} & \mathrm{P} 2 & 165 & \pm 35 & \mu \mathrm{M} \\ \mathrm{K}_{\mathrm{m} \text { (oleandomcyin) }} & \mathrm{P} 3 & 32 & \pm 8 & \mu \mathrm{M} \\ \mathrm{K}_{\mathrm{m} \text { (UDPG) }} & \mathrm{P} 4 & 36 & \pm 15 & \mu \mathrm{M}\end{array}$

Additional Table 3 Regression with the hypothesis of UDPGIc as A

Equation $Y=1 /\left(P 1 / A^{*} B /\left(P 2 * P 4+P{ }^{*} B+P 4 / A+B / A\right)\right)$

$\begin{array}{lllll}\mathrm{V}_{\max } & \mathrm{P} 1 & 0.00095 & \pm 0.00022 & \mathrm{mM} \\ \mathrm{K}_{\mathrm{I}(\text { UDPG })} & \mathrm{P} 2 & 182 & \pm 12 & \mu \mathrm{M} \\ \mathrm{K}_{\mathrm{m}(\text { UDPG })} & \mathrm{P} 3 & 36 & \pm 15 & \mu \mathrm{M} \\ \mathrm{K}_{\mathrm{m} \text { (oleandomcyin) }} & \mathrm{P} 4 & 32 & \pm 8 & \mu \mathrm{M}\end{array}$

Additional Figure 4 Lineweaver-Burke Plot for OleD with UDP5SGlc and Oleandomycin as substrates

(d) 


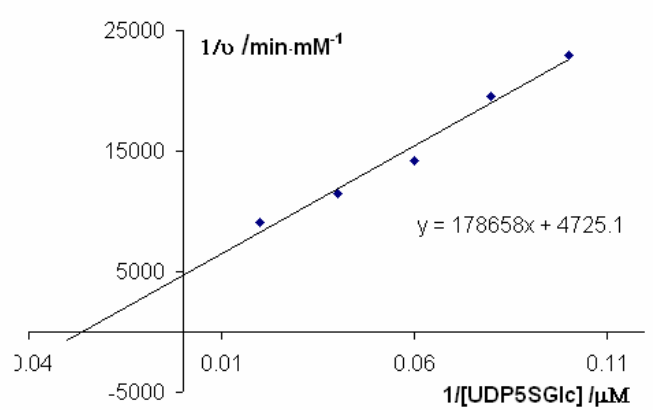

$\begin{array}{lll}\mathrm{V}_{\max } & 2.12 \mathrm{E}-4 & \mathrm{mM} \cdot \mathrm{min}^{-1} \\ \mathrm{~K}_{\mathrm{M}(\text { UDP5SG })} & 37.81 & \mu \mathrm{M} \\ \mathrm{k}_{\text {cat }}=\mathrm{Vmax}_{\mathrm{max}} / \mathrm{E}_{0} & 0.00328 & \mathrm{~s}^{-1} \\ \mathrm{k}_{\text {cat }} / \mathrm{K}_{\mathrm{M}} & & \\ \text { (UDP5SG) } & 86.70 & \mathrm{M}^{-1} \mathrm{~s}^{-1}\end{array}$

Additional Figure 5 Lineweaver-Burke Plot for Olel with UDPGIc and Oleandomycin as substrates

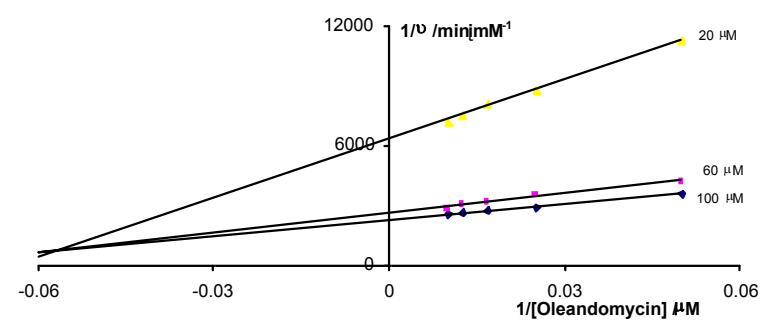

Additional Table 4 Regression with the hypothesis of Oleandomycin as $A$

Equation $Y=1 /\left(P 1 / A^{*} B /\left(P 2{ }^{*} P 4+P{ }^{*} B+P 4 / A+B / A\right)\right)$

$\begin{array}{lllll} & & & & \mathrm{mM} \\ \mathrm{V}_{\max } & \mathrm{P} 1 & 0.00093 & \pm 0.00004 & \mathrm{~min}^{-1} \\ \mathrm{~K}_{\mathrm{I} \text { (oleandomycin) }} & \mathrm{P} 2 & 18 & \pm 0.3 & \mu \mathrm{M} \\ \mathrm{K}_{\mathrm{M} \text { (oleandomcyin) }} & \mathrm{P} 3 & 4.8 & \pm 0.5 & \mu \mathrm{M} \\ \mathrm{K}_{\mathrm{M} \text { (UDPG) }} & \mathrm{P} 4 & 97 & \pm 6 & \mu \mathrm{M}\end{array}$

$\mathrm{K}_{\mathrm{M}(\mathrm{UDPG})} / \mathrm{K}_{\mathrm{M} \text { (oleandomcyin) }}=20$, indicates an Ordered $\mathrm{Bi} \mathrm{Bi}$ mechanism with the enzyme binding Oleandomycin first. ${ }^{[2]}$

$\begin{array}{lll}\mathrm{k}_{\text {cat }}=\mathrm{V}_{\text {max }} / \mathrm{E}_{0} & 0.042 & \mathrm{~s}^{-1} \\ \mathrm{k}_{\text {cat }} / \mathrm{K}_{\mathrm{M} \text { (Oleandomycin) }} & 8719 & \mathrm{M}^{-1} \mathrm{~s}^{-1} \\ \mathrm{k}_{\text {cat }} / \mathrm{K}_{\mathrm{M} \text { (UDPG) }} & 437 & \mathrm{M}^{-1} \mathrm{~s}^{-1}\end{array}$

Additional Figure 6 Lineweaver-Burke Plot for Olel with UDP5SGIc and Oleandomycin as substrates

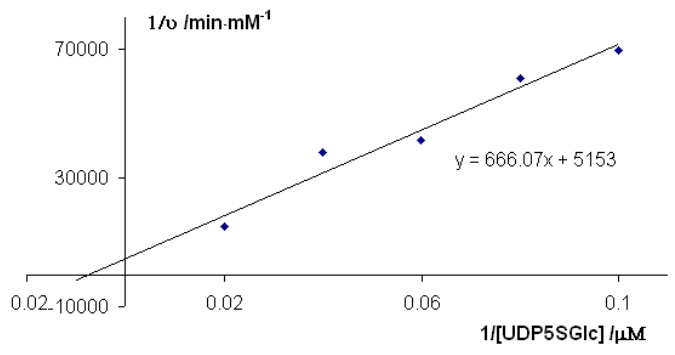

$\begin{array}{lll}\mathrm{V}_{\max } & 1.9 \mathrm{E}-4 & \mathrm{mM} \cdot \mathrm{min}^{-1} \\ \mathrm{~K}_{\mathrm{M}(\text { UDP5SG })} & 129 & \mu \mathrm{M} \\ \mathrm{K}_{\text {cat }}=\mathrm{Vmax}_{\text {mat }} & 0.013 & \mathrm{~s}^{-1} \\ \mathrm{k}_{\text {cat }} / \mathrm{K}_{\mathrm{M} \text { (UDP5SG) }} & 100 & \mathrm{M}^{-1} \mathrm{~s}^{-1}\end{array}$

Additional Figure 7 Lineweaver-Burke Plot for MGT with UDPGIc and Oleandomycin as substrates

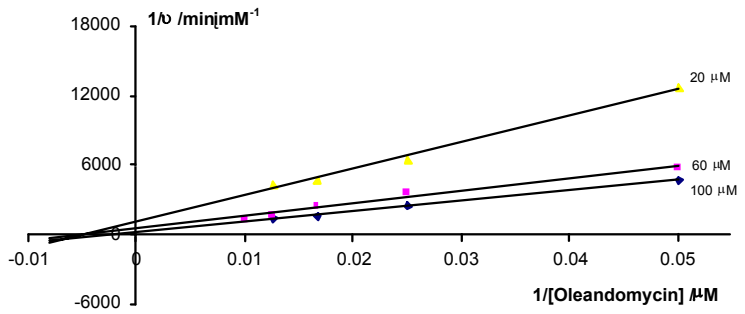

Intersection just in the $3^{\text {rd }}$ quadrant gives $\mathrm{K}_{\mathrm{IA}}<\mathrm{K}_{\mathrm{A}}$

Additional Table 5 Regression with the hypothesis of Oleandomycin as $\mathrm{A}$

Equation $\mathrm{Y}=1 /(\mathrm{P} 1 / \mathrm{A} * \mathrm{~B} /(\mathrm{P} 2 * \mathrm{P} 4+\mathrm{P} 3 * \mathrm{~B}+\mathrm{P} 4 / \mathrm{A}+\mathrm{B} / \mathrm{A}))$

$\begin{array}{lllll}\mathrm{V}_{\text {max }} & \mathrm{P} 1 & 0.024 & \pm 0.017 & \mathrm{mM}^{-1} \\ \mathrm{~K}_{\mathrm{I}(\text { oleandomycin) }} & \mathrm{P} 2 & 172 & \pm 8 & \mu \mathrm{M} \\ \mathrm{K}_{\mathrm{I}(\text { UDPG })} & \mathrm{P} 2 & 65 & \pm 0.4 & \mu \mathrm{M} \\ \mathrm{K}_{\mathrm{M} \text { (oleandomcyin) }} & \mathrm{P} 3 & 1305 & \pm 885 & \mu \mathrm{M} \\ \mathrm{K}_{\mathrm{M} \text { (UDPG) }} & \mathrm{P} 4 & 497 & \pm 354 & \mu \mathrm{M}\end{array}$

Additional Figure 8 Lineweaver-Burke Plot for MGT with UDP5SGIc and Oleandomycin as substrates

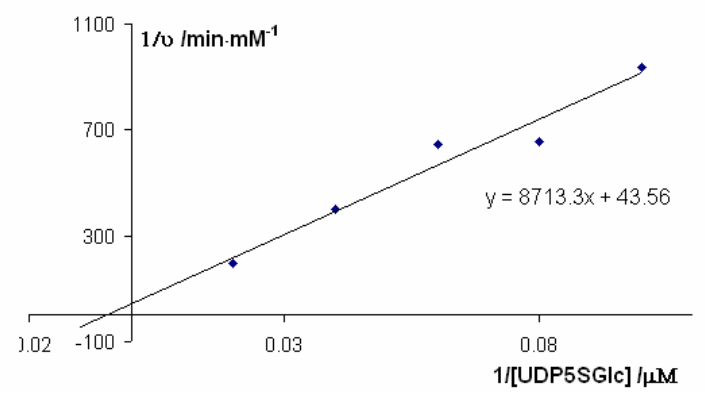

$\begin{array}{lll}\mathrm{V}_{\max } & 0.023 & \mathrm{mM} \cdot \mathrm{min}^{-1} \\ \mathrm{~K}_{\mathrm{M}(\text { UDP5SG })} & 200 & \mu \mathrm{M} \\ \mathrm{k}_{\text {cat }}=\mathrm{V}_{\text {max }} / \mathrm{E}_{0} & 1.8 & \mathrm{~s}^{-1} \\ \mathrm{k}_{\text {cat }} / \mathrm{K}_{\mathrm{M} \text { (UDP5SG) }} & 8990 & \mathrm{M}^{-1} \mathrm{~s}^{-1}\end{array}$

Additional Figure 9: Enzyme catalyzed synthesis of GalOleandomycin (1-Gal) and 5SGlc-Oleandomcyin (15SGIc)

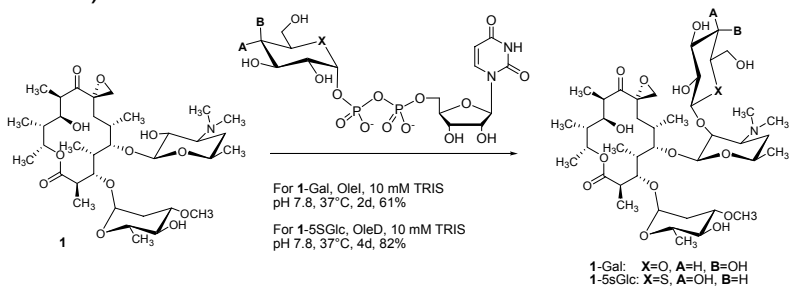




\section{Enzyme catalyzed synthesis of 1-Gal:}

UDPGal disodium salt (10.1 $\mathrm{mg}, 0.0166 \mathrm{mmol})$, Oleandomycin phosphate $(15.7 \mathrm{mg}, 0.02 \mathrm{mmol})$ and Olel $(5 \mathrm{mg} / \mathrm{mL}, 500 \mu \mathrm{L})$ were dissolved in TRIS buffer $(10 \mathrm{mM}$, $\mathrm{pH} 7.8,20 \mathrm{~mL}$ ) and incubated at $37^{\circ}$ for 2 days. The formation of product was monitored by MS. The reaction was terminated by heating to $90^{\circ} \mathrm{C}$ for $10 \mathrm{~min}$, centrifuged, the $\mathrm{pH}$ of supernatant adjusted to 10 and extracted with $\mathrm{CHCl}_{3}(20 \mathrm{~mL})$. The organic layer was reduced, $\mathrm{MeOH}$ $(0.5 \mathrm{~mL})$ added and purified by preparative TLC using $\mathrm{n}$ propanol : ethyl acetate : water : acetic acid (PEWA, $40: 31: 20: 1, \mathrm{v} / \mathrm{v} / \mathrm{v} / \mathrm{v})$ as the mobile phase. Bands at Rf 0.16 were collected to give 1-Gal $(8.5 \mathrm{mg}, 61 \%)$. ${ }^{1} \mathrm{H}$ NMR (400 $\mathrm{MHz}, \mathrm{CD}_{3} \mathrm{OD}$ ): $\delta=5.37$ (dq, $\left.J=1.7,6.4 \mathrm{~Hz}, 1 \mathrm{H}, \mathrm{H} 13\right)$, 4.97 (d, $\left.J=2.3 \mathrm{~Hz}, 1 \mathrm{H}, \mathrm{H} 1^{\prime \prime}\right), 4.40$ (d, J = 7.2 Hz, 1H, H1'), 4.38 (d, $\left.J=9.1 \mathrm{~Hz}, 1 \mathrm{H}, \mathrm{H} 1^{\prime \prime \prime}\right), 3.87(\mathrm{~d}, J=3.6 \mathrm{~Hz}, 1 \mathrm{H}$, H4"'), 3.85 (d, $J=4.6 \mathrm{~Hz}, 1 \mathrm{H}, \mathrm{H} 3$ ), 3.81 (dd, $J=4.0,7.1$ $\mathrm{Hz}, 1 \mathrm{H}, \mathrm{H6}$ '”), 3.80 (dd, J = 2.4, $4.6 \mathrm{~Hz}, 1 \mathrm{H}, \mathrm{H} 11$ ), 3.72 (m, $\left.1 \mathrm{H}, \mathrm{H} 5^{\prime \prime}\right), 3.70$ (m, 1H, H5'), 3.70 (dd, $J=4.5,9.4 \mathrm{~Hz}, 1 \mathrm{H}$, H6"'), 3.62 (t, J = 8.6 Hz, 1H, H2"'), $3.58(\mathrm{~m}, 1 \mathrm{H}, \mathrm{H} 5), 3.53$ (m, 1H, H5'"), 3.49 (m, 1H, H3"'), 3.48 (m, 1H, H2'), 3.45 (m, 1H, H3"), 3.45 (s, 3H, H7'), 3.20 (dd, $J=1.7,5.1 \mathrm{~Hz}$, $1 \mathrm{H}, \mathrm{H} 10), 3.05$ (t, $J=9.6 \mathrm{~Hz}, 1 \mathrm{H}, \mathrm{H} 4$ ”), 2.95 (m, 1H, H3'), $2.83(\mathrm{~d}, J=5.5 \mathrm{~Hz}, 1 \mathrm{H}, \mathrm{H} 18), 2.78(\mathrm{~d}, J=5.7 \mathrm{~Hz}, 1 \mathrm{H}$, $\mathrm{H} 18), 2.77(\mathrm{~m}, 1 \mathrm{H}, \mathrm{H} 2), 2.58$ (dd, $J=3.4,12.3 \mathrm{~Hz}, 1 \mathrm{H}$, $\mathrm{H} 7), 2.45$ (s, 6H, H7', 8'), $2.41\left(\mathrm{~m}, 1 \mathrm{H}, \mathrm{H} 2^{\prime \prime}\right), 1.86(\mathrm{~m}, 1 \mathrm{H}$, $\mathrm{H} 4), 1.92(\mathrm{~m}, 1 \mathrm{H}, \mathrm{H} 6), 1.90(\mathrm{~m}, 1 \mathrm{H}, \mathrm{H} 4), 1.67(\mathrm{t}, \mathrm{J}=7.6$ $\mathrm{Hz}, 1 \mathrm{H}, \mathrm{H} 12), 1.61$ (dd, $J=10.2,10.9 \mathrm{~Hz}, 1 \mathrm{H}, \mathrm{H} 7), 1.49$ (ddd, $J=3.7,13.0,13.3 \mathrm{~Hz}, 1 \mathrm{H}, \mathrm{H} 2$ '), 1.38 (m, 1H, H4'), $1.33(\mathrm{~d}, J=6.2 \mathrm{~Hz}, 3 \mathrm{H}, \mathrm{H} 14), 1.28(\mathrm{~d}, J=6.8 \mathrm{~Hz}, 3 \mathrm{H}$, H6”), 1.24 (d, $\left.J=5.4 \mathrm{~Hz}, 3 \mathrm{H}, \mathrm{H} 6^{\prime}\right), 1.23$ (d, $J=7.2 \mathrm{~Hz}, 3 \mathrm{H}$, $\mathrm{H} 16), 1.21$ (d, $J=6.8 \mathrm{~Hz}, 3 \mathrm{H}, \mathrm{H} 15), 1.18(\mathrm{~d}, J=6.5 \mathrm{~Hz}$, $3 \mathrm{H}, \mathrm{H} 17), 1.02$ (d, $J=6.9 \mathrm{~Hz}, 3 \mathrm{H}, \mathrm{H} 20), 1.00$ (d, $J=6.9$ $\mathrm{Hz}, 3 \mathrm{H}, \mathrm{H} 19) ;{ }^{13} \mathrm{C}$ NMR (100 MHz, CD $\left.\mathrm{OD}\right): \delta=209.5$ (C9), 179.1 (C1), 106.2 (C1'”), 102.5 (C1'), 98.5 (C1'”), 82.4 (C5), 81.5 (C2'), 79.5 (C3), 78.2 (C3”), 77.3 (C5”'), 77.3 (C4"), 74.3 (C3"'), 73.6 (C2'”), 72.2 (C13), 70.2 (C11), 69.8 (C5'), 69.5 (C5'), 68.2 (C4'”), 67.1 (C3'), 65.0 (C8), 61.5 (C6"'), 57.3 (C7'), 49.5 (C18), 46.3 (C10), 45.5 (C4), 45.2 (C2), 42.4 (C12), 39.5 (C7'), 39.5 (C8'), 34.9 (C2"), 34.0 (C6), 33.5 (C7), 30.0 (C4'), 21.2 (C6'), 19.2 (C17), 18.0 (C6"), 17.6 (C14), 12.5 (C15), 9.5 (C16), 7.5 (C19), 9.0 (C20); ${ }^{13} \mathrm{C}-{ }^{1} \mathrm{H}$ coupling constants from gradient phase sensitive HSQC: C1"-H1" $168.8 \mathrm{~Hz}$, indicated H1" is $\alpha$ linked; $\mathrm{C}^{\prime}-\mathrm{H} 1^{\prime} 155.6 \mathrm{~Hz}$ indicated $\mathrm{H} 1$ ' is $\beta$ linked;
C1"'-H1"' $150.5 \mathrm{~Hz}$ indicated $\mathrm{H} 1$ '"' is $\beta$ linked. HMBC, $\mathrm{H} 1$ '"' (4.38 ppm) coupled with C2' (81.5 ppm) confirmed galactosylation of O-2'. HRMS (ESI-) $\mathrm{m} / \mathrm{z} 884.4412$ ([M + $\mathrm{Cl}^{-}$) (calc. 884.4411).

\section{Enzyme catalyzed synthesis of 1-5SGIc:}

$\alpha$-UDP5SGIc $(0.0059 \mathrm{mmol})$, Oleandomycin phosphate (9.1 $\mathrm{mg}, 0.0119 \mathrm{mmol})$, OleD (8 $\mathrm{mg} / \mathrm{mL}, 2.5 \mathrm{~mL})$ were dissolved in MES buffer (1 mM, pH 6.5, $400 \mathrm{~mL})$ and incubated at $37{ }^{\circ} \mathrm{C}$ for $3 \mathrm{~d}$. Purification followed a very similar procedure to that described above except: extraction with $\mathrm{CHCl}_{3}(400 \mathrm{~mL})$ and the $\mathrm{Rf}$ of product 1$5 \mathrm{SGlc}$ is $0.24\left(30 \% \mathrm{MeOH}\right.$ in $\left.\mathrm{CHCl}_{3}\right)$. Note that it's slightly higher than Oleandomycin). Product obtained $(4.3 \mathrm{mg}$, $82 \%) .{ }^{1} \mathrm{H}$ NMR (400 MHz, $\left.\mathrm{CD}_{3} \mathrm{OD}\right): \delta=5.38$ (dq, $J=1.8$, $7.2 \mathrm{~Hz}, 1 \mathrm{H}, \mathrm{H} 13), 4.97$ (d, J = 2.7 Hz, 1H, H1"), 4.60 (d, J $=9.2 \mathrm{~Hz}, 1 \mathrm{H}, \mathrm{H} 1^{\prime \prime}$ ), 4.30 (d, J = $\left.7.9 \mathrm{~Hz}, 1 \mathrm{H}, \mathrm{H} 1^{\prime}\right), 4.01$ (dd, $J=3.7,10.9 \mathrm{~Hz}, 1 \mathrm{H}, \mathrm{H} 4$ '”), 3.88 (d, $J=6.7 \mathrm{~Hz}, 1 \mathrm{H}, \mathrm{H} 11)$, 3.81 (d, J = 5.3 Hz, 1H, H3), $3.69(\mathrm{~m}, 1 \mathrm{H}, \mathrm{H} 3$ '”), 3.67 (m, $1 \mathrm{H}, \mathrm{H} 5$ '), 3.65 (m, 1H, H6"'), 3.60 (t, J = 9.1 Hz, 1H, H2'”), $3.56\left(\mathrm{~m}, 1 \mathrm{H}, \mathrm{H} 5^{\prime}\right), 3.54$ (d, J = $\left.10.2 \mathrm{~Hz} 1 \mathrm{H}, \mathrm{H} 5\right), 3.44$ (m, $1 \mathrm{H}, \mathrm{H6}$ "') 3.43 (s, 3H, H7"), 3.43 (m, 1H, H3"), 3.30 (m, $\left.1 \mathrm{H}, \mathrm{H} 2^{\prime}\right), 3.21(\mathrm{~d}, J=6.0 \mathrm{~Hz}, 1 \mathrm{H}, \mathrm{H} 10), 3.04(\mathrm{t}, J=8.9 \mathrm{~Hz}$, $1 \mathrm{H}, \mathrm{H} 4$ "), 2.81 (m, 1H, H5"'), 2.81 (m, 1H, H18), 2.77 (m, $1 \mathrm{H}, \mathrm{H} 2), 2.77$ (m, Hz, 1H, H18), 2.77 (m, 1H, H3'), 2.62 (dd, $J=4.1,15.1 \mathrm{~Hz}, 1 \mathrm{H}, \mathrm{H} 7), 2.45$ (dd, $J=6.2,14.1 \mathrm{~Hz}$, $\left.1 \mathrm{H}, \mathrm{H} 2{ }^{\prime \prime}\right), 2.30$ (s, 6H, H7', 8'), $1.93(\mathrm{~m}, 1 \mathrm{H}, \mathrm{H} 4), 1.91$ (m, $1 \mathrm{H}, \mathrm{H} 6), 1.83\left(\mathrm{~m}, 1 \mathrm{H}, \mathrm{H} 4^{\prime}\right), 1.70$ (dd, $J=12.6,15.8 \mathrm{~Hz}$, $1 \mathrm{H}, \mathrm{H} 7), 1.65(\mathrm{~m}, 1 \mathrm{H}, \mathrm{H} 12), 1.44$ (ddd, $J=4.1,13.2,14.2$ $\mathrm{Hz}, 1 \mathrm{H}, \mathrm{H} 2$ "), 1.33 (d, J = 5.9 Hz, 3H, H14), $1.31(\mathrm{~m}, 1 \mathrm{H}$, H4'), 1.28 (d, $J=6.9 \mathrm{~Hz}, 3 \mathrm{H}, \mathrm{H} 16), 1.27$ (d, $J=6.2 \mathrm{~Hz}$, $\left.3 \mathrm{H}, \mathrm{H} 6^{\prime \prime}\right), 1.24$ (d, J = 6.8 Hz, 3H, H6'), 1.22 (d, J = 6.8 Hz, $3 \mathrm{H}, \mathrm{H} 17), 1.17$ (d, $J=7.3 \mathrm{~Hz}, 3 \mathrm{H}, \mathrm{H} 15), 1.03$ (d, $J=6.6$ $\mathrm{Hz}, 3 \mathrm{H}, \mathrm{H} 20), 1.01$ (d, $J=6.2 \mathrm{~Hz}, 3 \mathrm{H}, \mathrm{H} 19) ;{ }^{13} \mathrm{C} N M R$ (100 MHz, CD $\left.{ }_{3} \mathrm{OD}\right): \delta=209.3$ (C9), 179.5 (C1), 101.8 (C1'), 99.7 (C1'), 82.5 (C2'), 82.4 (C1'”), 81.8 (C5), 79.5 (C3), 79.2 (C2'”), 79.1 (C4"), 78.2 (C3”), 72.3 C13), 70.6 (C11), 70.0 (C5"), 69.5 (C6"') 69.1 (C5'), 66.1(C3'), 65.1 (C8), 62.0 (C4"'), 61.9 (C3"'), 57.3 (C7"), 49.3 (C18), 47.3 (C5"'), 46.4 (C10), 45.8 (C2), 45.2 (C4), 42.3 (C12), 39.7 (C7'), 39.7 (C8'), 35.0 (C2"), 33.1 (C6), 32.8 (C7), 29.9 (C4'), 21.3 (C6'), 19.2 (C17), 18.1 (C6”), 17.8 (C14), 13.1 (C15), 9.6 (C16), 9.2 (C20), 7.3 (C19);MS (ESI-); HMBC, H1'" (4.60 ppm) coupled with C2' (82.5 ppm) confirmed thioglucosylation of O-2'. HRMS $\mathrm{m} / \mathrm{z} 900.4174$ ([M + $\left.\left.\mathrm{Cl}^{-}\right]^{-}\right)$, (calc. 900.4182).

Additional Table 6: ${ }^{1} \mathrm{H}$ and ${ }^{13} \mathrm{C}$ NMR data of glycosylated oleandomycins 1-Gal and 1-5SGlc in $\mathrm{CD}_{3} \mathrm{OD}$.
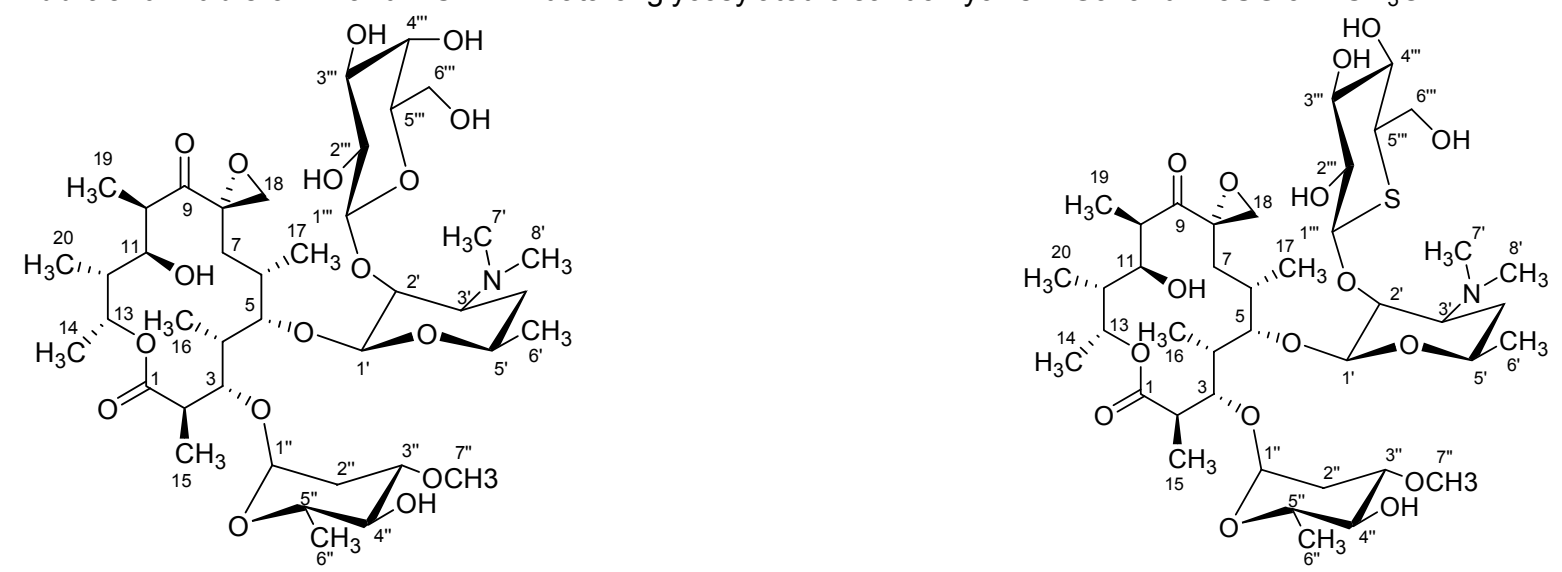


\begin{tabular}{|c|c|c|c|c|c|c|c|c|}
\hline \multicolumn{5}{|l|}{ 1-Gal } & \multicolumn{4}{|l|}{ 1-5SGIc } \\
\hline Site & ${ }^{13} \mathrm{C} / p p m$ & $\begin{array}{l}{ }^{1} \mathrm{H} \\
\text { Ippm }\end{array}$ & Multiplicity & $J(\mathrm{H}, \mathrm{H})(\mathrm{Hz})$ & ${ }^{13} \mathrm{C} / p p m$ & $\begin{array}{l}{ }^{1} \mathrm{H} \\
\text { Ippm }\end{array}$ & Multiplicity & $J(\mathrm{H}, \mathrm{H})(\mathrm{Hz})$ \\
\hline 1 & 179.1 & - & & & 179.5 & - & & \\
\hline 2 & 45.2 & 2.77 & $\mathrm{~m}$ & & 45.8 & 2.77 & $\mathrm{~m}$ & \\
\hline 3 & 79.5 & 3.85 & $d$ & 4.6 & 79.5 & 3.81 & $d$ & 5.3 \\
\hline 4 & 45.5 & 1.86 & $\mathrm{~m}$ & & 45.2 & 1.93 & $\mathrm{~m}$ & \\
\hline 5 & 82.4 & 3.58 & $\mathrm{~m}$ & & 81.8 & 3.54 & $\mathrm{~d}$ & 10.2 \\
\hline 6 & 34.0 & 1.92 & $\mathrm{~m}$ & & 33.1 & 1.91 & $\mathrm{~m}$ & \\
\hline \multirow[t]{2}{*}{7} & 33.5 & 1.61 & dd & $10.2,10.9$ & 32.8 & 1.77 & dd & $12.6,15.8$ \\
\hline & & 2.58 & dd & $3.4,12.3$ & & 2.62 & dd & $4.1,15.1$ \\
\hline 8 & 65.0 & - & & & 65.1 & - & & \\
\hline 9 & 209.5 & - & & & 209.3 & - & & \\
\hline 10 & 46.3 & 3.20 & dd & $1.7,5.1$ & 46.4 & 3.21 & $d$ & 6.0 \\
\hline 11 & 70.2 & 3.80 & dd & $2.4,4.6$ & 70.6 & 3.88 & $d$ & 6.7 \\
\hline 12 & 42.4 & 1.67 & $t$ & 7.6 & 42.3 & 1.65 & $\mathrm{~m}$ & \\
\hline 13 & 72.2 & 5.37 & $\mathrm{dq}$ & $1.7,6.4$ & 72.3 & 3.58 & $\mathrm{dq}$ & $1.8,7.2$ \\
\hline 14 & 17.6 & 1.33 & $d$ & 6.2 & 17.8 & 1.33 & $d$ & 5.9 \\
\hline 15 & 12.5 & 1.21 & $d$ & 6.8 & 13.1 & 1.17 & $d$ & 7.3 \\
\hline 16 & 9.5 & 1.23 & $d$ & 7.2 & 9.6 & 1.28 & $d$ & 6.9 \\
\hline 17 & 19.2 & 1.18 & d & 6.5 & 19.2 & 1.22 & $d$ & 6.8 \\
\hline \multirow[t]{2}{*}{18} & 49.5 & 2.78 & $\mathrm{~d}$ & 5.7 & 49.3 & 2.77 & $\mathrm{~m}$ & \\
\hline & & 2.83 & $d$ & 5.5 & & 2.81 & $\mathrm{~m}$ & \\
\hline 19 & 7.5 & 1.00 & $d$ & 6.9 & 7.3 & 1.01 & $d$ & 6.2 \\
\hline 20 & 9.0 & 1.02 & $d$ & 6.9 & 9.2 & 1.03 & $d$ & 6.6 \\
\hline $1^{\prime}$ & 102.5 & 4.40 & $\mathrm{~d}$ & 7.2 & 101.8 & 4.30 & $\mathrm{~d}$ & 7.9 \\
\hline $2^{\prime}$ & 81.5 & 3.48 & $\mathrm{~m}$ & & 82.5 & 3.30 & $\mathrm{~m}$ & \\
\hline $3^{\prime}$ & 67.1 & 2.95 & $\mathrm{~m}$ & & 66.1 & 2.77 & $\mathrm{~m}$ & \\
\hline \multirow[t]{2}{*}{$4^{\prime}$} & 30.0 & 1.38 & $\mathrm{~m}$ & & 29.9 & 1.31 & $\mathrm{~m}$ & \\
\hline & & 1.90 & $\mathrm{~m}$ & & & 1.83 & $\mathrm{~m}$ & \\
\hline $5^{\prime}$ & 69.5 & 3.70 & $\mathrm{~m}$ & & 69.1 & 3.56 & $\mathrm{~m}$ & \\
\hline $6^{\prime}$ & 21.2 & 1.24 & $d$ & 5.4 & 21.3 & 1.24 & $d$ & 6.8 \\
\hline 7', 8' & 39.5 & 2.45 & $S$ & & 39.7 & 2.30 & $\mathrm{~s}$ & \\
\hline 1" & 98.5 & 4.97 & d & 2.3 & 99.7 & 4.97 & $d$ & 2.7 \\
\hline \multirow[t]{2}{*}{$2 "$} & 34.9 & 1.49 & ddd & $3.7,13.0,13.3$ & 35.0 & 1.44 & ddd & $4.1,13.2,14.2$ \\
\hline & & 2.41 & $\mathrm{~m}$ & & & 2.45 & dd & $6.2,14.1$ \\
\hline 3" & 78.2 & 3.45 & $\mathrm{~m}$ & & 78.2 & 3.43 & $\mathrm{~m}$ & \\
\hline $4 "$ & 77.3 & 3.05 & $t$ & 9.6 & 79.1 & 3.04 & $\mathrm{t}$ & 8.9 \\
\hline 5" & 69.8 & 3.72 & $\mathrm{~m}$ & & 70.0 & 3.67 & $\mathrm{~m}$ & \\
\hline 6" & 18.0 & 1.28 & d & 6.8 & 18.1 & 1.27 & $\mathrm{~d}$ & 6.2 \\
\hline 7" & 57.3 & 3.45 & $\mathrm{~s}$ & & 57.3 & 3.43 & $\mathrm{~s}$ & \\
\hline $1 "$ & 106.2 & 4.38 & $d$ & 9.1 & 82.4 & 4.60 & $d$ & 9.2 \\
\hline $2^{\prime \prime \prime}$ & 73.6 & 3.62 & $\mathrm{t}$ & 8.6 & 79.2 & 3.60 & $\mathrm{t}$ & 9.1 \\
\hline 3 & 74.3 & 3.49 & $\mathrm{~m}$ & & 61.9 & 3.69 & $\mathrm{~m}$ & \\
\hline $4 "$ & 68.2 & 3.87 & $d$ & 3.6 & 62.0 & 4.01 & dd & 3.7 .10 .9 \\
\hline 5 & 77.3 & 3.53 & $\mathrm{~m}$ & & 47.3 & 2.81 & $\mathrm{~m}$ & \\
\hline \multirow[t]{2}{*}{$6 "$} & 61.5 & 3.70 & dd & $4.5,9.4$ & 69.5 & 3.44 & $\mathrm{~m}$ & \\
\hline & & 3.81 & $\mathrm{dd}$ & $4.0,7.1$ & & 3.65 & $\mathrm{~m}$ & \\
\hline
\end{tabular}


Additional Figure 10. Acceptor and donor library

a) Acceptor library

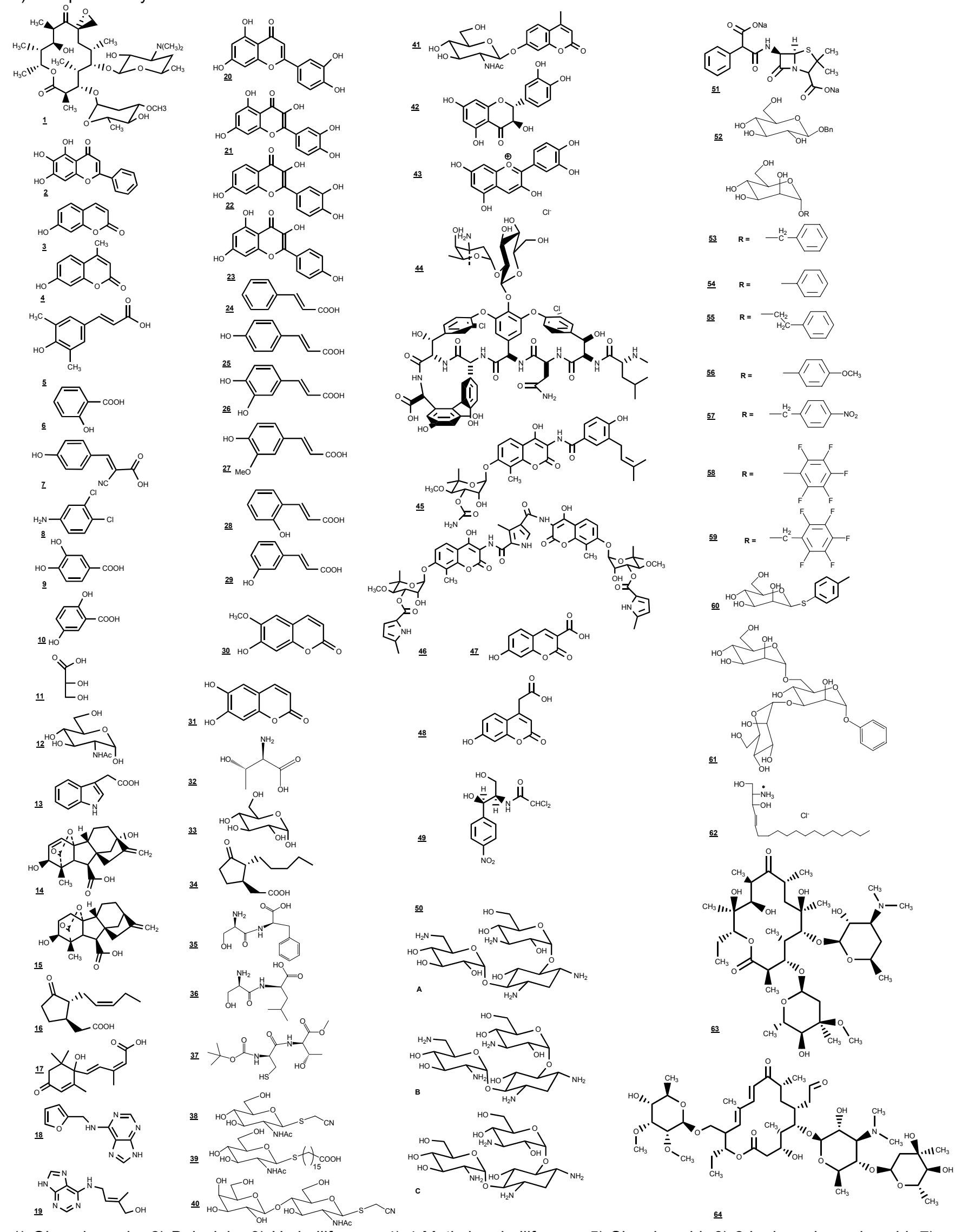

1) Oleandomycin, 2) Baicalein, 3) Umbelliferone, 4) 4-Methyl-umbelliferone, 5) Sinapic acid, 6) 2-hydroxy-benzoic acid, 7) acyano-4-hydroxyl-cinamic acid, 8) 3,4-dichloroaniline, 9) 3,4-dihydroxylbenzoic acid, 10) 2,5-dihydroxylbenzoic acid, 11) D-

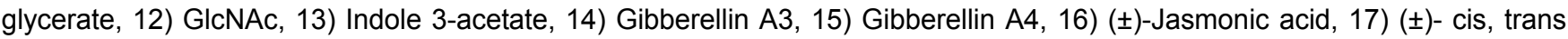


Abscisic acid, 18) Kinetin, 19) Zeatin, 20) Luteolin, 21) Quercetin, 22) Fisetin, 23) Kaempferol, 24) Cinnamic acid, 25) 4hydroxy cinnamic acid, 26) 3,4-dihyroxy cinnamic acid, 27) 4-hydroxy 3-methoxy cinnamic acid, 28) 2-hydroxy cinnamic acid, 29) 3-hydroxy cinnamic acid, 30) 7-hydroxy 6-methoxy coumarin (Scopoletin), 31) 6,7-dihydroxy coumarin (Esculetin), 32) Threonine, 33) Glucose, 34) Dihydrojasmonic acid, 35) Ser-Phe 36) Ser-Leu, 37) BocCysThrOMe, 38) 1-Thio-S-cyanomethyl$\mathrm{N}$-acetyl-D-glucosamine, 39) 1-Thio-S-(16-hexadecanoic acid)-N-acetyl-D-glucosamine, 40) 1-Thio-S-cyanomethyl- $N$-acetyl- $D$ lactosamine, 41) MUGIcNAc, 42) Trans-dihydroquercetin (DHQ), 43) Cyanidin chloride (CYN), 44) Vancomycin, 45) Novobiocin, 46) Coumermycin A1, 47) 7-hydroxycoumerin 3-carboxylic acid, 48) 7-hydroxycoumerin 4-acetic acid, 49) Chloramphenicol, 50) Kanamycin, 51) Carbencillin disodium salt, 52) B-GlcOBn, 53) $\alpha-M a n O B n, ~ 54) \alpha-M a n O P h, ~ 55) ~ \alpha-$

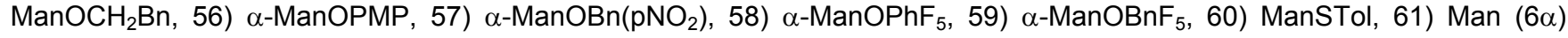
$\operatorname{Man}(3 \alpha) \operatorname{Man}(\alpha) \mathrm{OPh}, 62) D$-erythro-spingosine, 63) Erythromycin, 64) Tylosin

b) Donor library

$$
\text { 每 }
$$

A: $\alpha$-UDP-D-Glucose

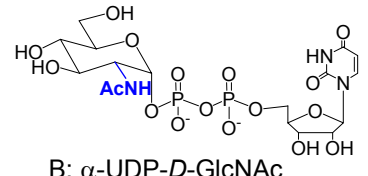

B: $\alpha$-UDP-D-GICNAc

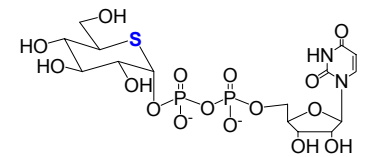

C: $\alpha$-UDP-5-thio- $D$-glucose

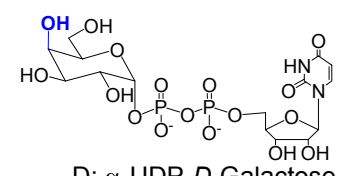

D: $\alpha$-UDP-D-Galactose

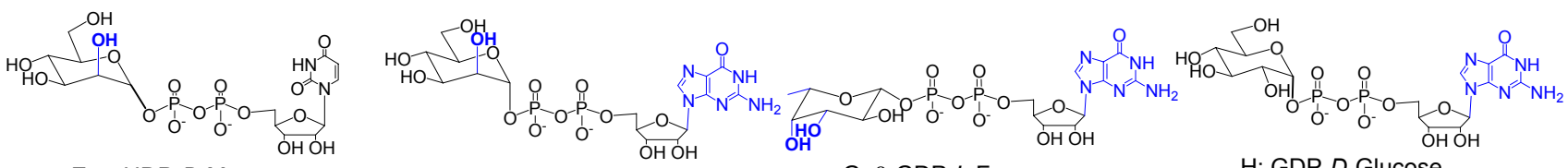

E: $\alpha$-UDP-D-Mannose

F: $\alpha$-GDP-D-Mannose

G: $\beta$-GDP-L-Fucose

H: GDP-D-Glucose

$$
\mathrm{OH}_{\mathrm{OH}}^{\mathrm{O}}
$$

$$
\underbrace{-O^{2}}
$$

M: UDP-3-deoxy-3-

fluoro- $D$-Galactose

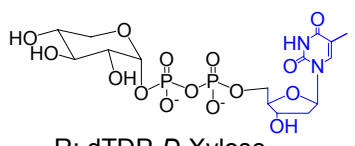

R: dTDP-D-Xylose

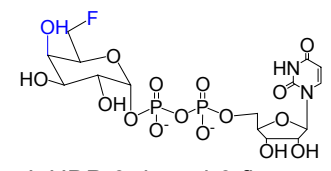

J: UDP-6-deoxyl-6-fluoro$D$-Galactose

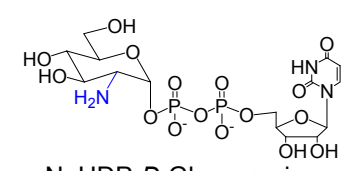

$\mathrm{N}$ : UDP-D-Glucosamine

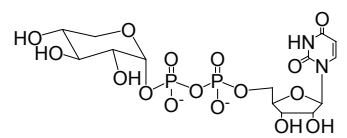

S: UDP-D-Xylose

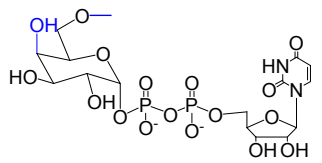

K: UDP-6-O-methyl- $D$ Galactose

$$
\underbrace{-O^{2}}
$$

P: UDP-2-deoxy-2fluoro- $D$-Galactose

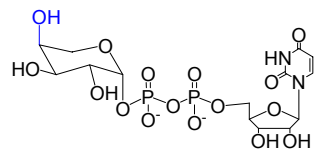

L: UDP-L-Arabinose

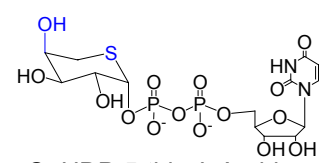

Q: UDP-5-thio-L-Arabinose

Additional Figure 11: GAR results of UDPGIc and library acceptors using (a) OleD (b) Olel (c) MGT. Green means positive result, amber means unclear and red means negative.

11(a)

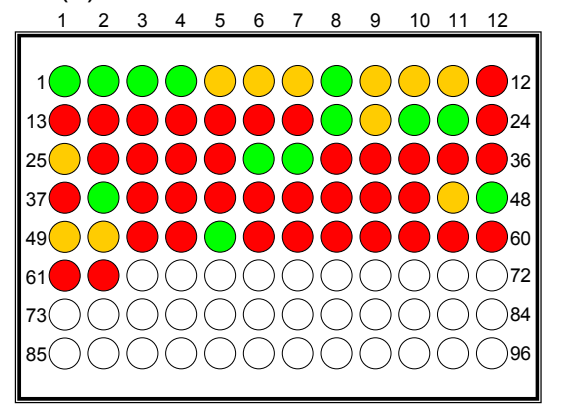




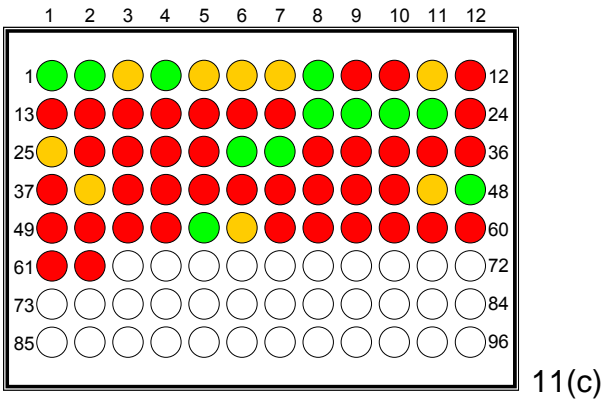

Additional Figure 12: GAR results of varied donors with selected acceptors using (a) OleD (b) Olel (c) MGT
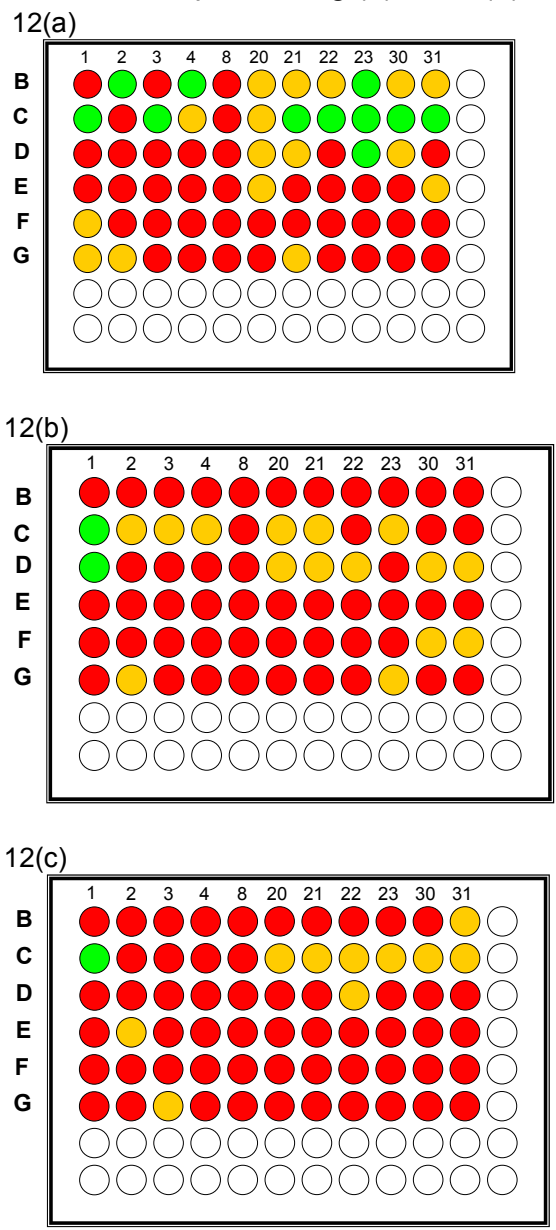

Additional Figure 13: GAR results of donor library with (a) Oleandomycin using OleD, Olel and MGT; (b) Erythromycin or Tylosin using OleD and MGT

13(a)

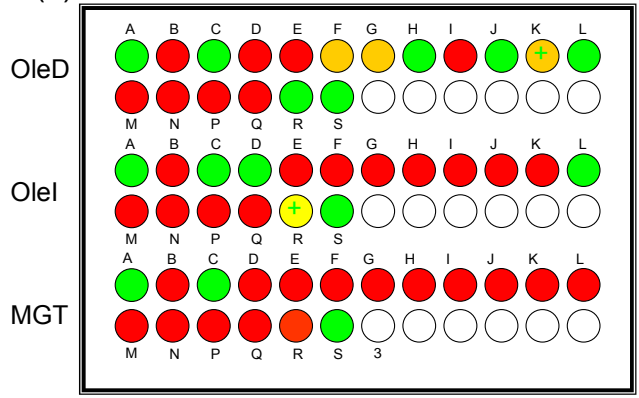

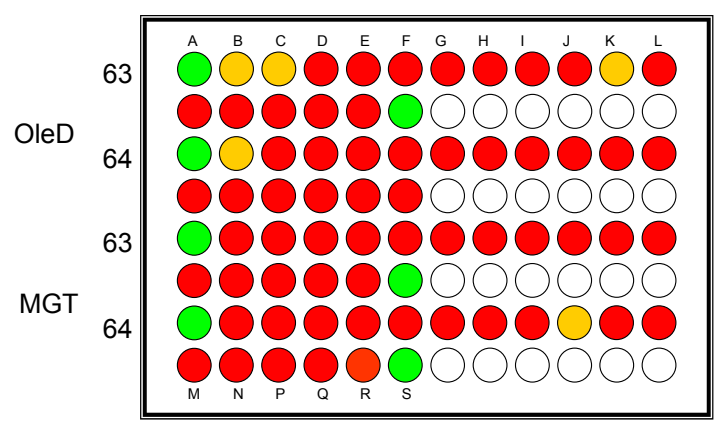

$13(b)$

All the enzymes are restricted in the C2 and C3 donor configuration, but tolerate the change of ring oxygen to sulfur. MGT and Olel cannot (or very weakly) use other donor bases rather than uridine, indicating that the donor binding pocket of MGT and Olel are quite similar to each other but different to OleD, which uses $U, G$ and TDP sugars. OleD also tolerates changes on $\mathrm{C} 6$ and $\mathrm{C} 4$.

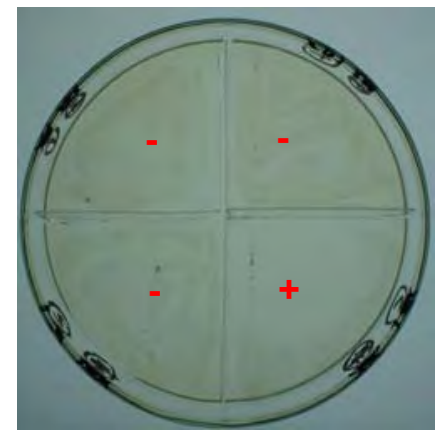

Additional Fig 15: (a) An LB-agar plate containing Kanamycin $50 \mu \mathrm{g} / \mathrm{ml}$ was divided into 4 parts (as above). Top left contains Oleandomycin $(50 \mu \mathrm{g} / \mathrm{ml})$, bottom left contains 1-Glc $(50 \mu \mathrm{g} / \mathrm{ml})$, top right contains $1-\mathrm{Gal}$ (50 $\mu \mathrm{g} / \mathrm{ml})$ and bottom right contains 1-Gal $(50 \mu \mathrm{g} / \mathrm{ml})$ and IPTG (1 mM). E. Coli. BL21 was transformed with $\beta$ galactosidase DNA (Ss $\beta G)$ overnight media $(5 \mu \mathrm{L})$ was plated on each part and incubated at $37^{\circ} \mathrm{C}$ for overnight. The bottom right area showed inhibited growth of cells.

(b) 1-Gal uptake test by BL21

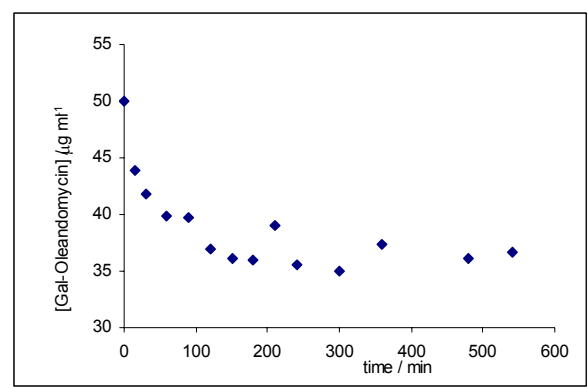

A $25 \mathrm{ml} \mathrm{Ss} \beta \mathrm{G}$ overnight cell culture was harvested by centrifuge $\left(3000 \times \mathrm{g}, 4^{\circ} \mathrm{C}, 30 \mathrm{~min}\right)$ and resuspended in TRIS buffer ( $5 \mathrm{ml}, 10 \mathrm{mM}, \mathrm{pH} 7.0)$. Cells were starved by shaking at $37^{\circ} \mathrm{C}, 200 \times \mathrm{g}$ for 2 hours and Gal-Ole was added to make the final concentration of $50 \mu \mathrm{g} / \mathrm{ml}$ and then the shake continued. Aliquots $(20 \mu \mathrm{L})$ were taken out every 15 or $30 \mathrm{~min}$. Each aliquot was added to EtOH (20 $\mu \mathrm{L})$ and centrifuged $(14000 \times \mathrm{g}, 10 \mathrm{~min})$ to remove cells. Supernatant $(30 \mu \mathrm{L})$ was mixed with TRIS buffer $(30 \mu \mathrm{L})$ and analyzed by LC/MS using the method reported ${ }^{[3]}$. 
After $540 \mathrm{~min}$, cell media $(2 \mathrm{ml})$ was centrifuged (14000 $x \mathrm{~g}, 10 \mathrm{~min}$ ) and cells were washed carefully with TRIS buffer $(3 \times 1 \mathrm{ml})$ to remove any contamination. Cells were harvested, resuspended in $0.5 \mathrm{ml}$ TRIS buffer and broken by sonication $\left(3 \times 1 \mathrm{~min}, 0^{\circ} \mathrm{C}\right)$. Supernatant was obtained by centrifuge and analyzed by LC/MS. This figure (b) shows the disappearance of $1-\mathrm{Gal}$ from the solution. Cell extraction at $540 \mathrm{~min}$ shows that there was intracellular 1Gal equal to $14 \mu \mathrm{g} / \mathrm{ml}$ while from the average extracelluar loss in the above figure, $13.5 \mu \mathrm{g} / \mathrm{ml}$ was expected.

(c) 1-Gal uptake test by Tuner.

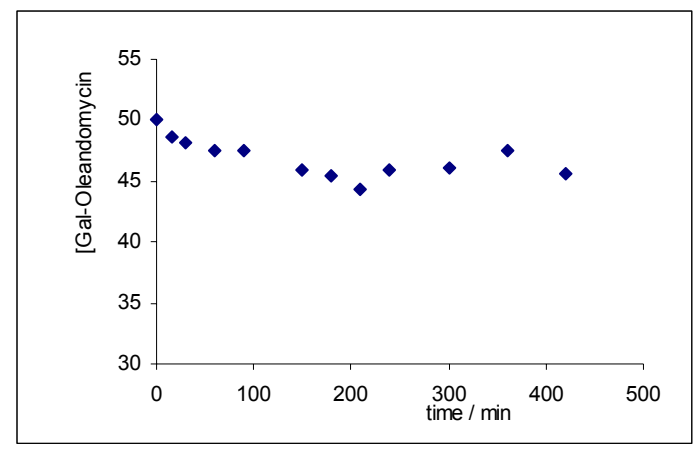

Experiment was conducted in the same way as above using Tuner.

\section{Additional References}

[1] B. Miroux, J. E. Walker, J. Mol. Biol. 1996, 260, 289.

[2] M. Yang, M. Brazier, R. Edwards, B. G. Davis, ChemBioChem 2005, 6, 346-357.

[3] M. Yang, PhD Thesis, University of Huddersfield (UK), 2003. 